\title{
Research Paper \\ Effect of Family-Based Empowerment Model on the Self-Efficacy of Hypertensive Elderly People

\section{${ }^{*}$ Batool Hedayati ${ }^{1}$}

1. Department of Community Health, School of Nursing and Midwifery, Isfahan University of Medical Sciences, Isfahan, Iran.

Received: 07 Aug. 2017

Accepted: 18 Nov. 2017

Key words:

Empowerment,

Family-centered,

Self-efficacy, Elderly,

Hypertension
Citation: Hedayati B. [Effect of Family-Based Empowerment Model on the Self-Efficacy of Hypertensive Elderly People (Persian)]. Iranian Journal of Ageing. 2018; 13(1):86-97. https://doi.org/10.21859/SIJA.13.1.86

dol: : https://doi.org/10.21859/SIJA.13.1.86

\begin{abstract}
A B S T R A C T
Objectives Non-communicable diseases are important factors of mortality and morbidity in the elderly people. Among them, hypertension is the most common cardiovascular disease. The family-centered empowerment model is considered to be an important model for promoting health. Thus, the aim of this study was to evaluate the effect of the model on self-efficacy in elderly people with hypertension. Methods \& Materials This clinical trial study was conducted on 62 elderly people with hypertension in the Lenjan health center. After sampling by randomized clustering, the subjects were assigned to experimental and control groups. The family-centered empowerment model was used in the experimental group while the control group had one educational class. The research tools were demographic check list and dimension of self-efficacy of empowerment questionnaire. Post-test was carried out 1 week after the intervention and was followed up after 1.5 months. The obtained data were analyzed by means of SPSS20 Software and Dedicational Statistical Tests, Mann-Whitney, Chi-Square, T-Test, and ANOVAS.

Results Before the intervention, both groups were matched with respect to the means of self-efficacy dimension score $(P>0.05)$; but 1 week and 1.5 months after the intervention, significant differences were observed in experimental group $(P<0.05)$.

Conclusion In this study, the family-centered empowerment model showed an effect on the self-efficacy of elderly people with hypertension.
\end{abstract}

\section{Extended Abstract}

\section{Objective}



ging population is a phenomenon that has been observed since the twentieth century [1]. This age group mainly suffers from non-communicable diseases, including high blood pressure, and, hence, is considered as one of the main consumers of health services [2]. It has been reported that the promotion of self-efficacy is effective in preventing, reducing, and controlling diseases [5]. Health promotion indicates that the family role is central in the process of preventing and treating diseases [11]. Methods to help individuals and their families to have an active role in their health care must emphasize on empowering them [14]. Family-centered empowerment model

\section{* Corresponding Author:}

Batool Hedayati, MSc.

Address: Department of Community Health, School of Nursing and Midwifery, Isfahan University of Medical Sciences, Isfahan, Iran.

Tel: +98 (913) 1371558

E-mail: parastaran2011@yahoo.com 
is designed to improve the effectiveness of the role of the individual and family members and is aimed at empowering the patients in their illness [16]. The current study has been conducted with the aim of determining the effect of family-centered empowerment model on the self-efficacy of hypertensive elderly individuals.

\section{Methods and Materials}

This randomized clinical trial study was conducted with two-group and three-stage designs and with pretest and post-test on 62 hypertensive elderly people. After receiving the letter of introduction from the Vice-Chancellor for Research of Isfahan Nursing and Midwifery Faculty and written permission (with the code of 392189), the researcher referred to the Lanjan Healthcare Network. Using cluster sampling, the samples were divided into two groups of experimental and control. The inclusion criteria were 60 years and older, having a high blood pressure of above 140/90 as approved and registered by the doctor of a health center, having a health record at the center, willingness to participate in the research, completion of Empowerment Dimension Inventory, and obtaining a score of a medium or weak in the scale.

Exclusion criteria included the unwillingness of the elderly to continue cooperation in the empowerment plan, unwillingness to complete the research tools, and not attending meetings more than once. For the experimental group, the family-centered empowerment model was based on the pattern of steps. The first step was the increase in threat understanding through group discussions. The second step was self-efficacy through group discussion and practical presentation. The third step was promoting self-esteem through the transfer of learned material by the elderly to a family member. The fourth step was evaluation involving process evaluation and the final evaluation. Empow- erment was performed in the form of questions and answers during the intervention sessions and as group discussions in the final stage. Common care and a training session were conducted for the control group. Research instruments were Demographic Information Checklist, the Empowerment Questionnaire, and Measurement of Self-efficacy Dimension. The posttest evaluation was conducted a week later, and it was followed-up a month and a half later. Independent ttest, analysis of variance, and SPSS20 software were used for data analysis.

\section{Results}

The results of this study showed that there was no significant difference between the two groups in the mean scores of self-efficacy feeling before the intervention $(\mathrm{P}<0.05)$. This was expected due to the random allocation of samples and no interventions. But within a week and a month and a half after the intervention, there was a significant difference between the two groups $(\mathrm{P}<0.001)$, and the elderly self-esteem increased (Table 1). This may be because promoting knowledge and awareness on high blood pressure and the benefits of its treatment increases self-efficacy. According to the description, signs and serious side effects of this disease that cannot be treated, providing practical solutions and creating self-belief and empowerment by using this pattern, had led to a significant increase in self-efficacy and hope in the elderly [6]. Empowerment programs, which include physical, family-centered and community-based activities, will significantly help the elderly to be self-efficacious and empowered [7]. Therefore, the importance of familycentered empowerment model in old age is prioritized because old age is a stage in people's lives that makes them exposed to chronic diseases including high blood pressure [6].

Table 1. Comparison of the mean score of elderly empowerment in feeling of self-efficacy in two groups of experimental and control at different times

\begin{tabular}{cccc}
\hline Time & \multicolumn{3}{c}{ Independent T-Test } \\
\cline { 2 - 4 } & $\mathbf{t}$ & $\mathbf{P}$ \\
\hline Before intervention & 1.1 & 0.275 \\
One week after intervention & 12.63 & $<0.001$ & $<0.001$ \\
A month and half after intervention & 12.6 & 1.12 & 0.341 \\
Analysis of variance with repeated observation & $\mathrm{F}$ & 11.15 & $<$ \\
\end{tabular}


The elderly use their own skills and resources to enhance their self-efficacy experience. In this study, it seems that with empowerment intervention and increasing the awareness and mastery of the elderly on some skills such as stress control skills, the skill of measuring blood pressure, self-efficacy has been improved in them [8]. By paying attention to the nature of aging and high blood pressure, the family members and health personnel can improve their self-efficacy. Many of their problems can be prevented if the elderly are well-informed about proper nutrition, exercise, sleep, relaxation, and proper use of medications. Moreover, these problems can be controlled effectively, and measures can be taken to improve the life quality of the elderly [9].

\section{Conclusion}

Based on the results of this study and the importance of self-efficacy in improving the health of the elderly, it is recommended that workshops focusing on the family-centric empowerment model be held by health authorities so as to improve the self-efficacy of the elderly. Empowerment leads to control of the individual's environment, self-efficacy, and self-esteem. Intrapersonal structures as a set of people's beliefs, feeling of the care provider, and interacting with family lead to self-control, self-efficacy, and self-sufficiency. This is the first study on the use of family-centered empowerment model in the hypertensive elderly. In fact, limited study time and working with elderly people were the limitations of this study.

\section{Acknowledgments}

This research was extracted from the MSc. thesis of the author in the Department of Community Health, School of Nursing and Midwifery, Isfahan University of Medical Sciences, Iran.

\section{Conflict of Interest}

The authors declared no conflicts of interest. 


\title{
تأثير اجراى الكَوى توانمندسازى خانوادهمحور برخودكار آمدى سالمندان با فشار خون بالا
}

\author{
"بتول هدايتى'

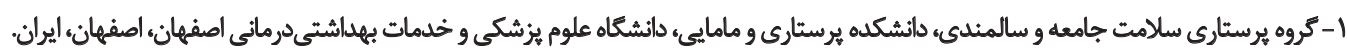

\section{حكSי}

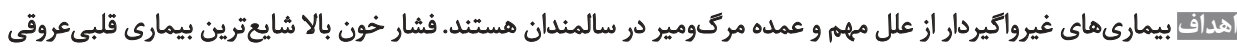

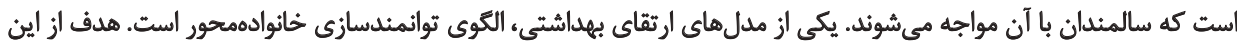

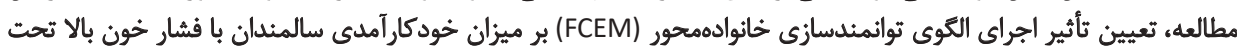

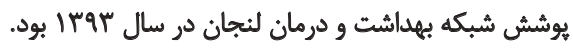
















يالا شده است.
تاريخ دريافت: 19 مرداد تاريخ بذيرش: Tr آبان









بيمارىهاي غيرواكيردار يكى از معضلات اصلى اين كروه








[ه] كه در دوران كهن خطالى شيوع فراوان دارد. تعداد بيماران



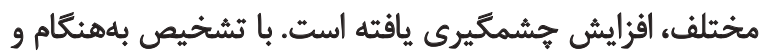





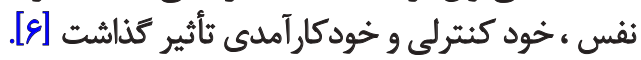























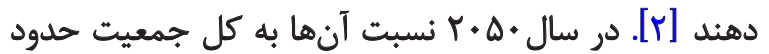


ارتقاى خود كار آمدى در بيشغيرى، كاهش وكنترل بيمارىهاى سالمندان به علت مشكلات فراوان سلامتى، بيشترين 


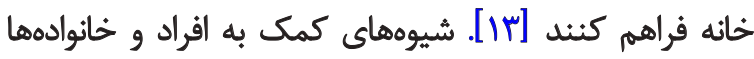

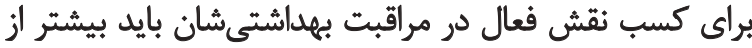

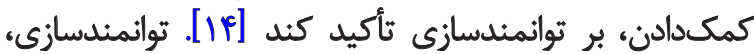

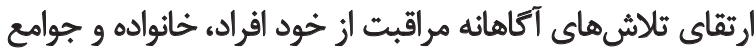

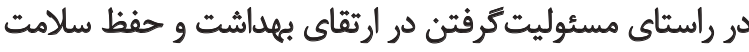

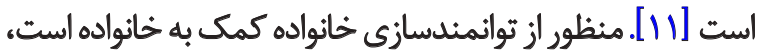

به نحوى كه بتواند به تغيير برسد [1/إن]

القوى توانمندسازى خانوادهمحور التويى بومى است كه با باديا

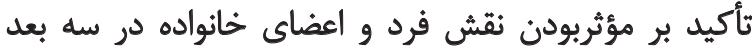

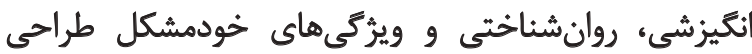



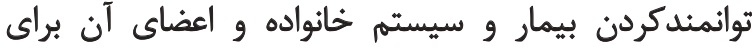

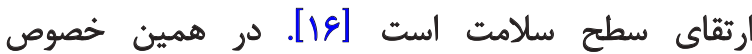

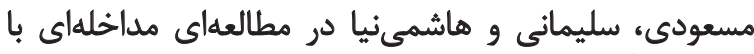

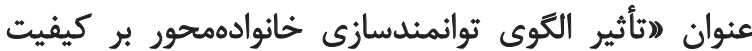

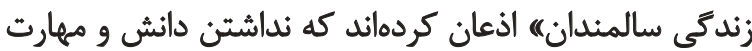



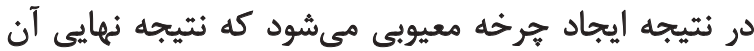

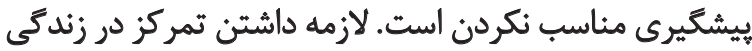



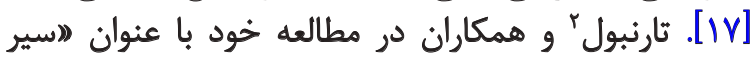

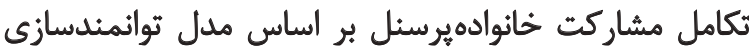

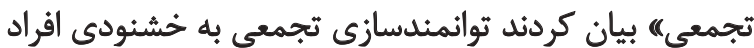

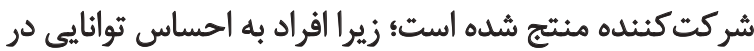

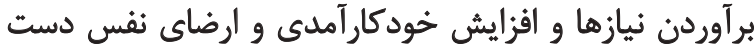

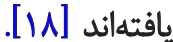

با توجه به مراجعات مكرر سالمندان مبتلا به فشار خون

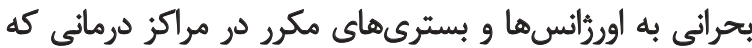

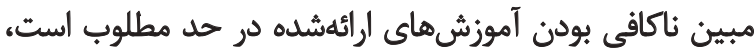

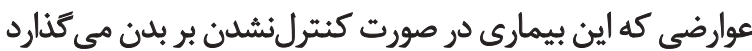

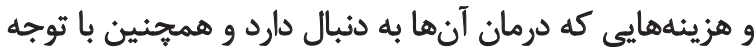

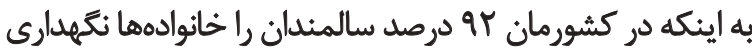

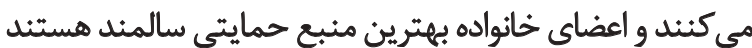

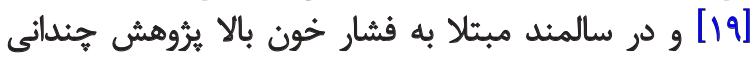

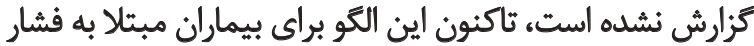

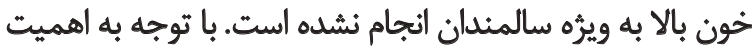

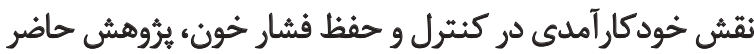

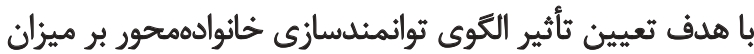

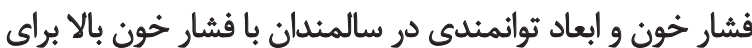

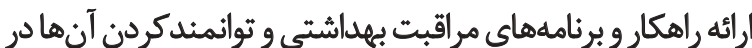

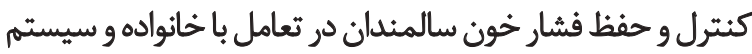

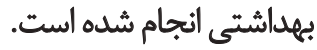

روحىروانى مؤثر است؛ در نتيجه با سلامت روان افراد ارتباط

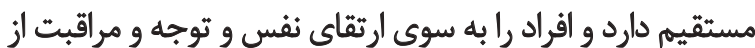

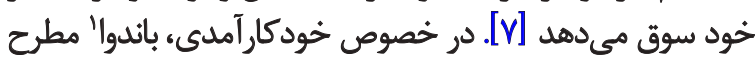

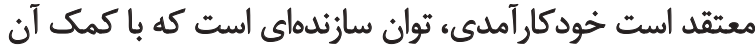

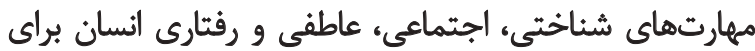

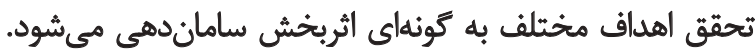

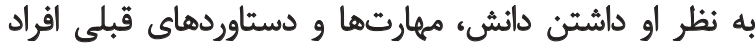

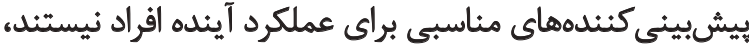

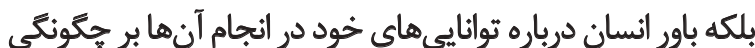

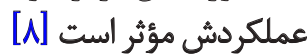

عقايد مرتبط با خودكارآمدى، بر اهداف و آرزوها اثر مى كذارند

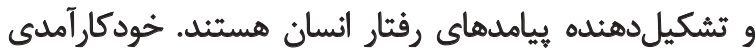

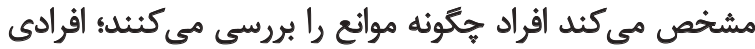



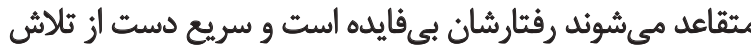

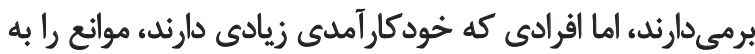


برابر مشكلات ايستادكى مي كنئد ويكمن در تعريف باورهاى خودكارآمدى بيان مىكني



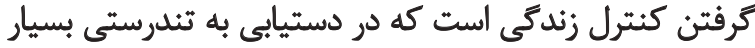

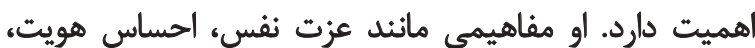

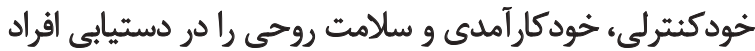

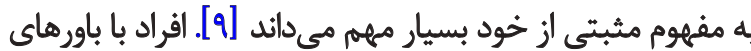

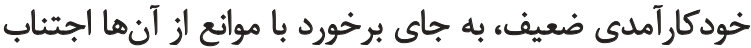

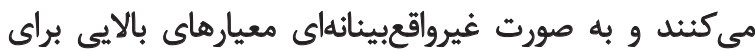

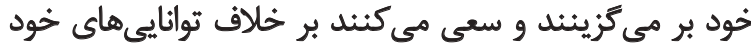

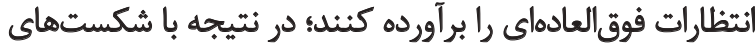

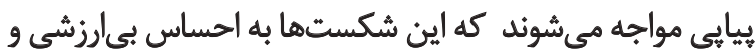

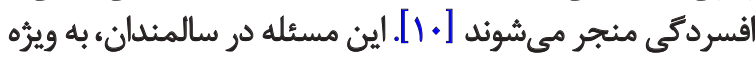

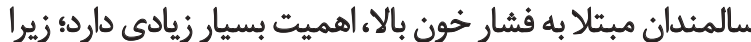

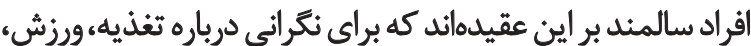

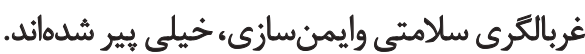
ارتقاى بهداشت حاكى از آن است كه در فرايندهاى بيشعَيرى

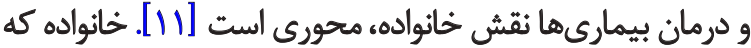

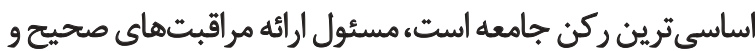

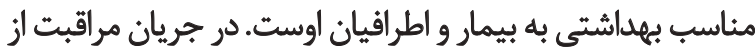

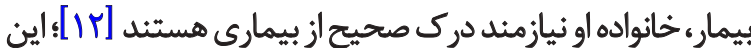

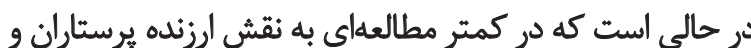

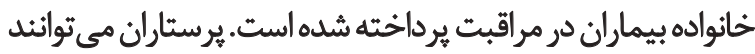
دانش، مهارت و حمايت لازم را براي حفظ كيفيت مراقبت درافيت درانيت 


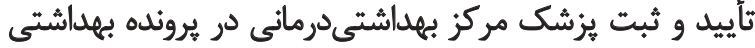

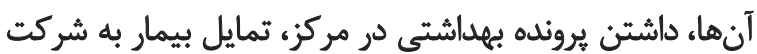

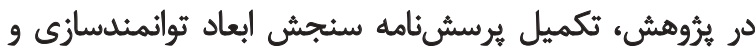

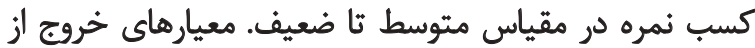

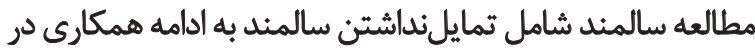

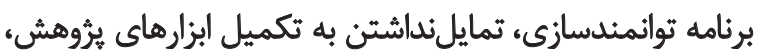

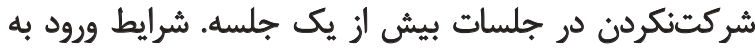

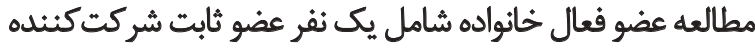

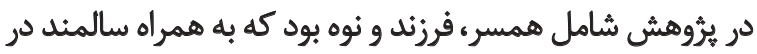

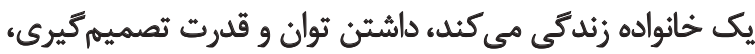

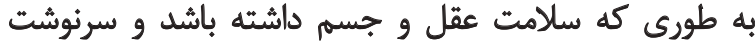

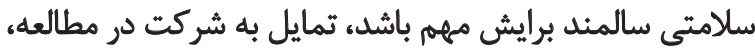

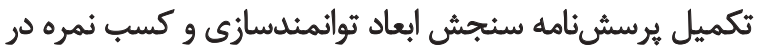

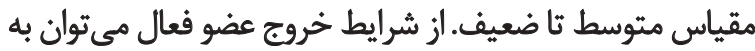

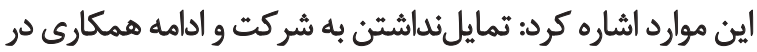

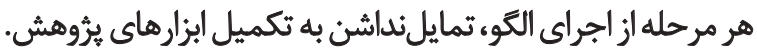

يس از اتمام نمونهيرى، سالمندان مدنظر در مركز

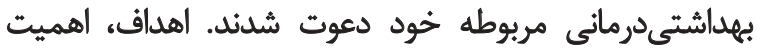

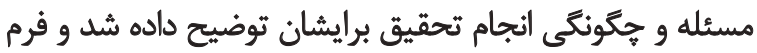

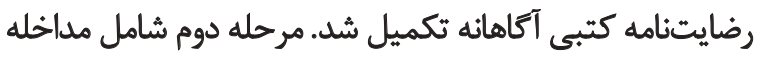

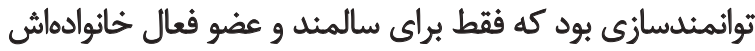

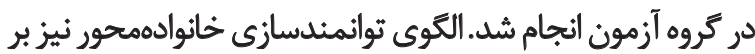

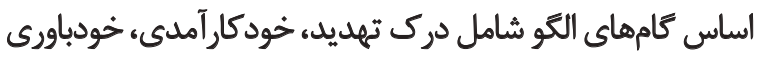
و ارزشيابي به ترتيب الجراشد. كام اول: درك تهبديد اولين مرحله در الكوى توانمندسازى خانوادهمحور افزايش

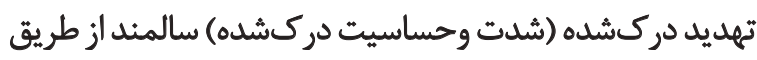

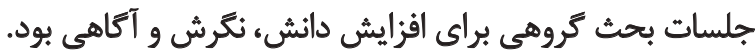

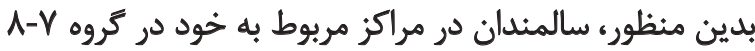

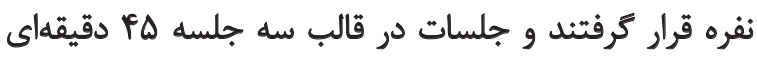

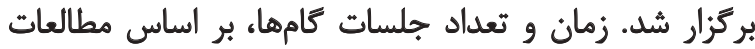

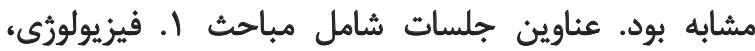

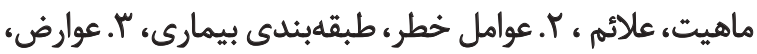

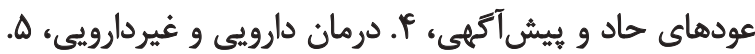

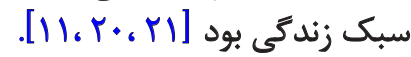

$$
\text { كام دوم: حُودكارآملدى }
$$

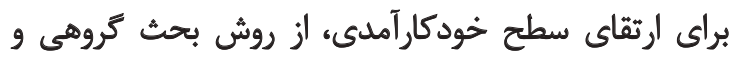

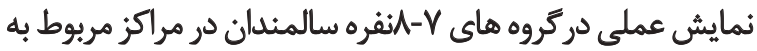

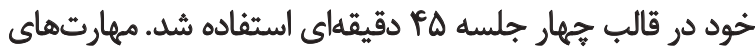

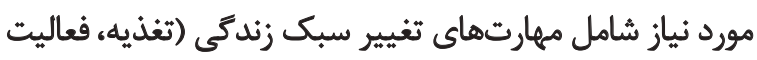

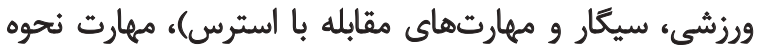

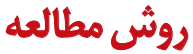

اين مطالعه كار آزمايي بالينى شاهدار تصادفى شده، به صورت

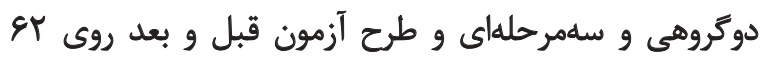

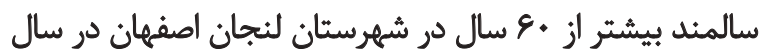

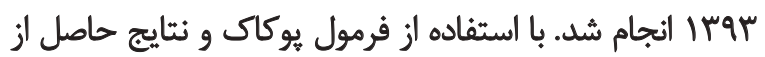

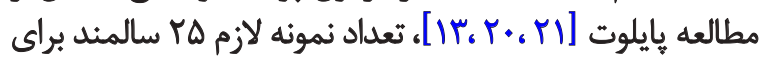

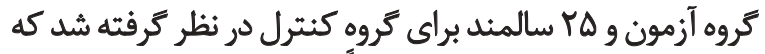

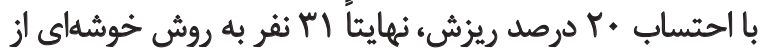

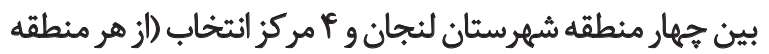
يك مركز) براى هر كروه برآورد شد.

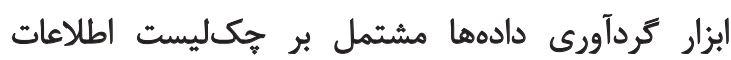

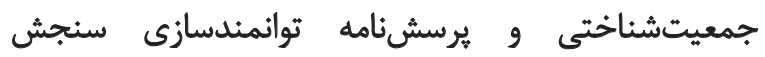

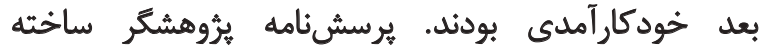

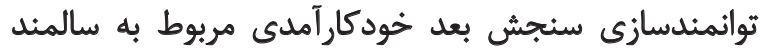

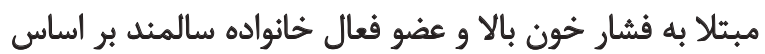

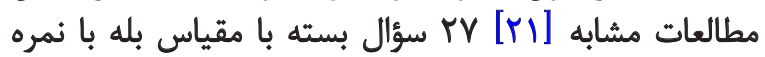

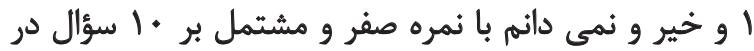

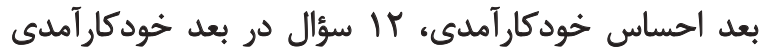

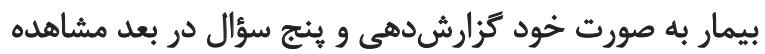

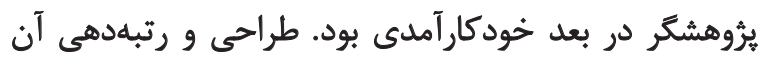

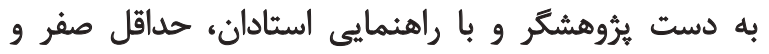

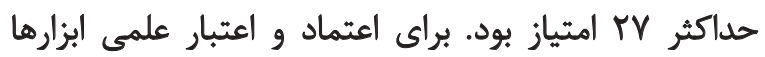

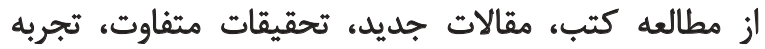

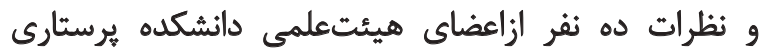

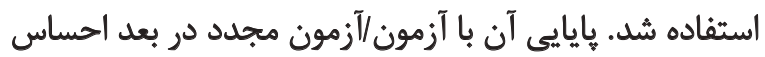

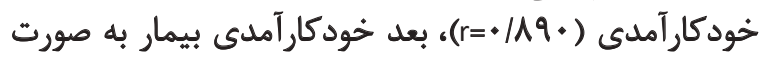

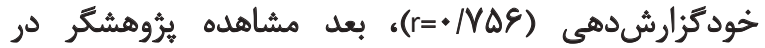

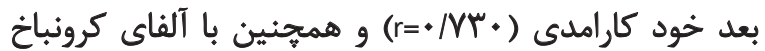

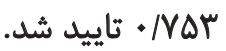

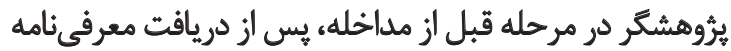

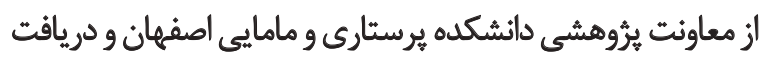

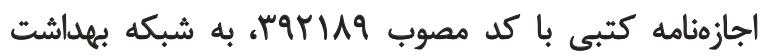

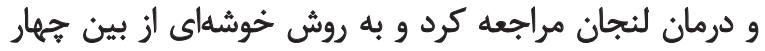

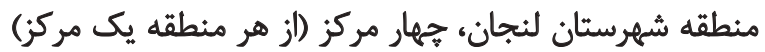

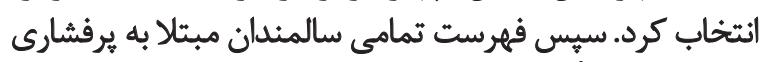

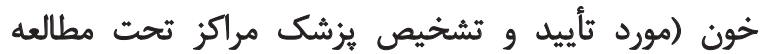

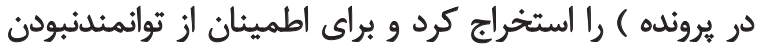

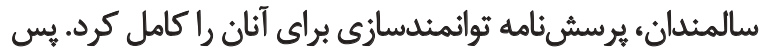

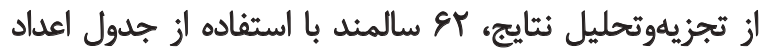
تصادفى به دو كروه آزمون و كنترل تخصيص داعند داده شدند.

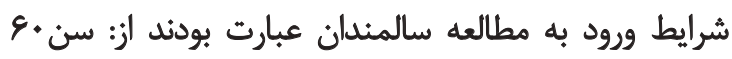

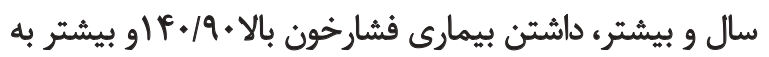


توانماسيقهاي ديكر و با تكميل يرسشنامه سنجش ابعاد

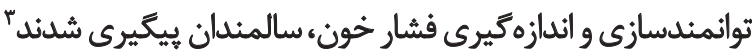
(ارزشيابى نهايى).

هي إز بايان كام سوم (البته اين از محدوديتهاي مطالعه

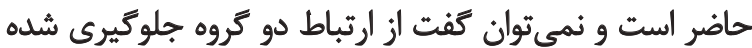

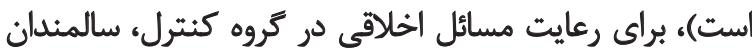

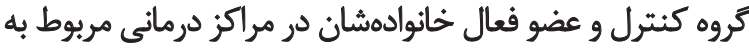

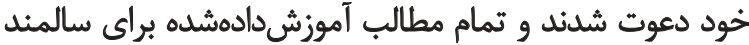

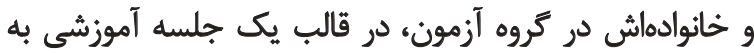

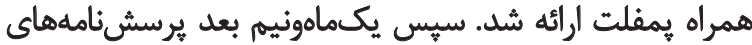

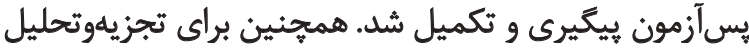

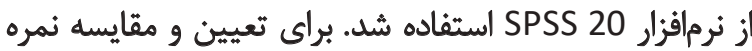

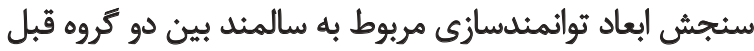

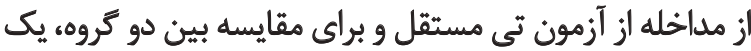

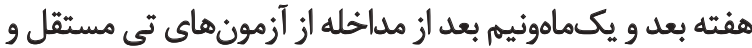

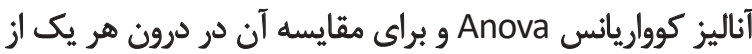

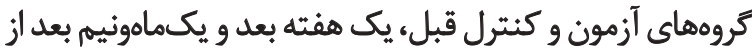

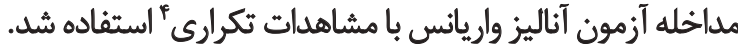

يافتهها

نتايج اين بيروهش نشان داد در كروهائ سالمندان و عضو

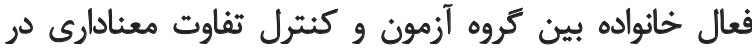

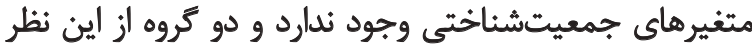

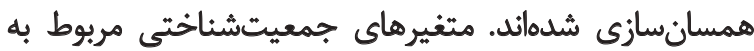

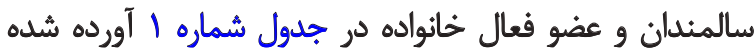

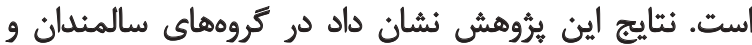

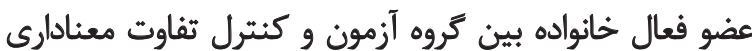

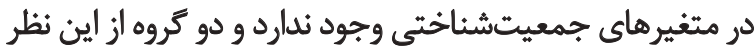

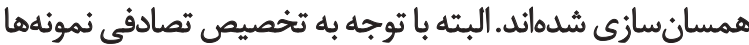

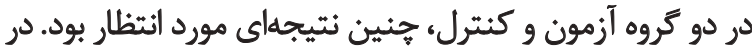

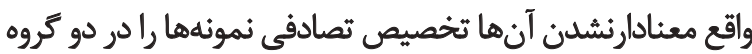

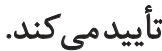

بحث

در مطالعه حاضر تأثير اجراى الخوى توانمندسازى خانوادهمحور

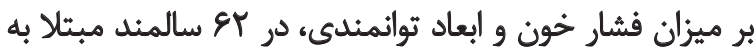

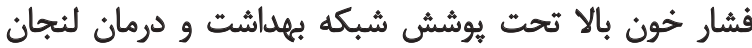

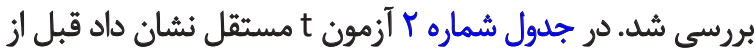

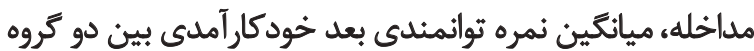

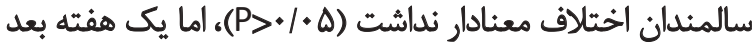

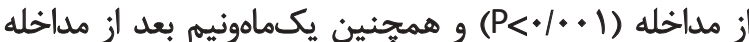

3. Follow up

4. Repeated measure anova

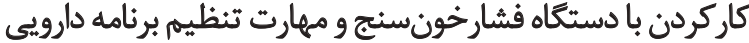

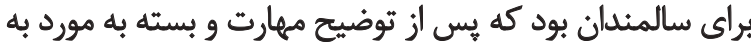

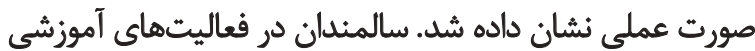

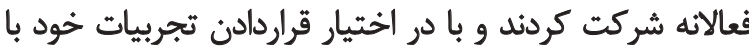

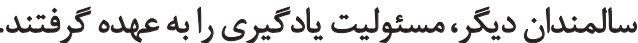

كام سوم: خودباورى

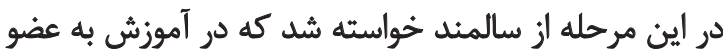

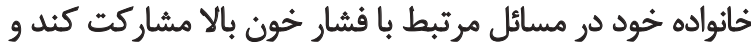

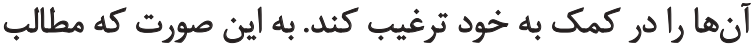

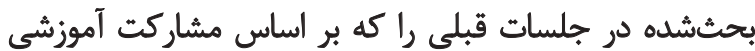

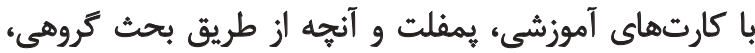

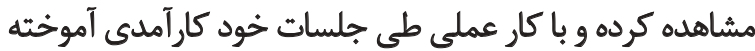

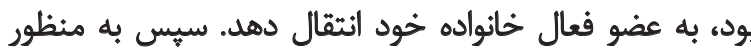

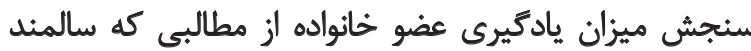

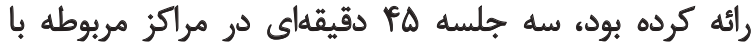

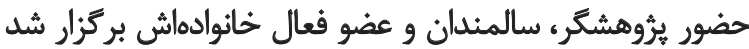

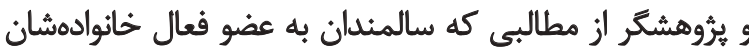

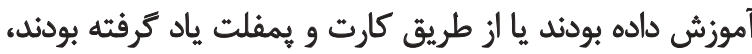
بازخورد دريافت كرد. كام همهارم: اروزشيابى

كام همهارم شامل ارزشيابى فرايند و ارزشيابى نهايي بود:

\section{ارزشيابى فرايند}

طى جلسات مداخله توانمندى به صورت برسشورئاسخ و بحث

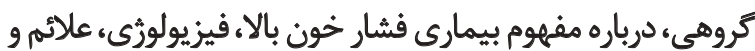

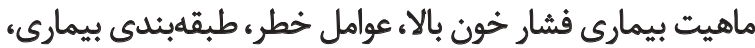

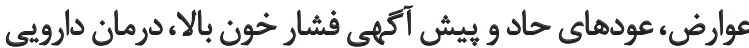

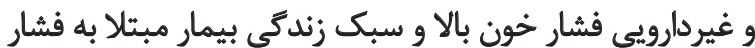

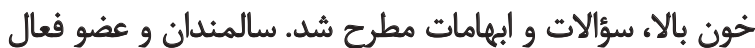

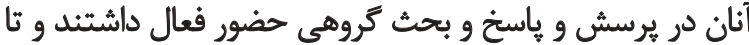
رفع ابهام جلسه ادامه داشت.

بعد از طىكردن كامهاى الكو، يُوهشكر براى اطمينان

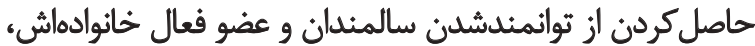

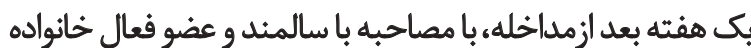

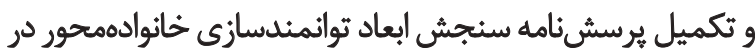

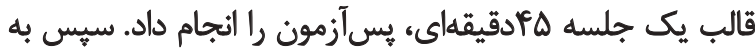

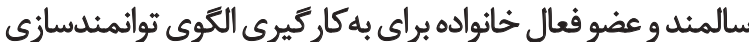
خانوادهمحور فرصت صاده شيد.

ارزشيابى نهايى(مرحله بعد از مداخله) سرانجام يكماهونيم بعد از مداخله و در قالب يك جلسه 
جدول ا. تعيين و مقايسه توزيع قراوانى متغيرهاي دموكرافيك سالمثد

\begin{tabular}{|c|c|c|c|c|}
\hline \multicolumn{4}{|c|}{$\mathbf{P}$} & \multirow{2}{*}{ متغير } \\
\hline عضو فعال خانواده & سالمند & عضو فعال خانواده & سالمند & \\
\hline $1 \cdot 18$ &.$/ 9 T^{\prime} \wedge$ & $r+/ q \pm \varepsilon 1 . q$ & $\varepsilon \& / q \pm \Delta / \Delta$ & سن سن \\
\hline.$/ T V A$ & ./9Tा & $\theta / / \Delta \pm 11 / \cdot \Delta$ & ge/TEIr & وزن \\
\hline.$/ 148$ &.$/ 119$ & $18 \Delta / V \pm \mathscr{V} \cdot r$ & $109 / Y \pm N \cdot r$ & قلد \\
\hline.$/ M M$ &.$/ \mathrm{ra}$ & $M r / r \pm \Psi / 81$ & $r \Delta / A \pm T / Q$ & BMI \\
\hline.$/ 1 F A$ & 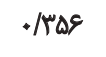 & - & - & سطح تحصيلات \\
\hline .1 .98 &. trife & - - & $\longrightarrow$ & منبع درآمد \\
\hline$+|8|$ & . WE & - & - & جنس \\
\hline.$|r \Lambda|$ & $.11 N$ & - & - & 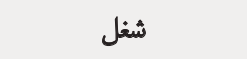 \\
\hline- & $+11 \Delta \Delta$ & - & $N V \pm \Delta / r$ & سال هاى ابتالا \\
\hline- & - MITF & - & $\longrightarrow$ & سابقه بيمارىهاى ديكر \\
\hline- & . Mres & - & $\Longrightarrow$ & سابقه دارودرمائي \\
\hline.$/ 1 v e$ & - - & - & - & نسبت خانوادكى با سالمند \\
\hline.$/ 1 Y T A$ & - & - - & - & تعلفاد اعضاي خانواده \\
\hline$+/ 94$ & -—- & - & $\longrightarrow$ & مرحله تكاملى خانواده \\
\hline
\end{tabular}

ill

ميانكين بعد خودكارآمدى بين دو كروه عضو فعال خانواده

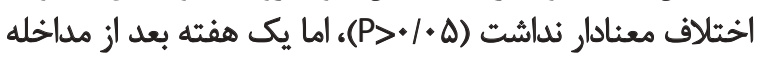

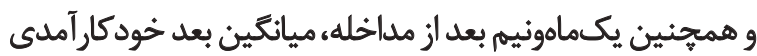

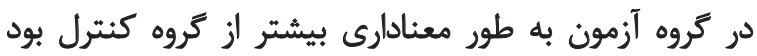

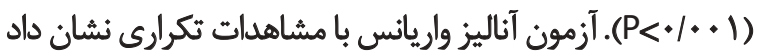

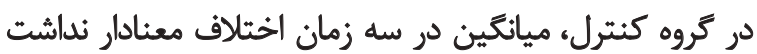

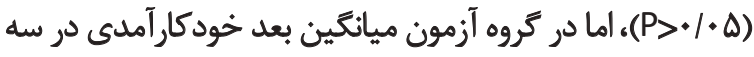

ميانكين نمره توانمندى بعد خود كارآمدى در كروه

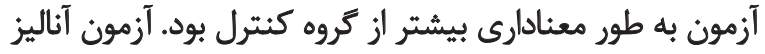

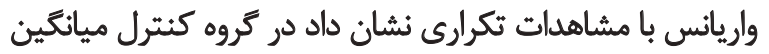

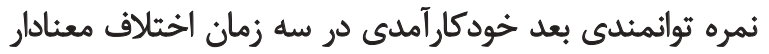

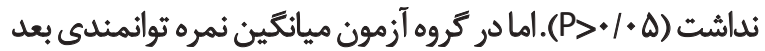

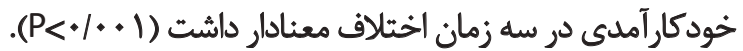
در جدول شماره r آزمون t مستقل نشان داد قبل از مداخله،

جدول r. مثايسه ميائكين نمره توائمثدى سالمندان در بعد احساس خودكارآمدى در دو تروه آزمون و كنترل در زمانهاى مختلف

\begin{tabular}{|c|c|c|c|}
\hline \multicolumn{2}{|c|}{ أزمون t مستقل } & & \multirow{2}{*}{ ز } \\
\hline $\mathbf{P}$ & $\mathbf{t}$ & & \\
\hline.$/ M V \Delta$ & $M$ & & قبيل از مداخله \\
\hline$<\cdot 1 \cdot .1$ & $\mid r / q \pi$ & & يك هفته بعل از مداخله \\
\hline$<+1 .+1$ & IYle & & يك ماه و نيه بعل از مداخله \\
\hline $\begin{array}{l}\text { WH } \\
. / M F I\end{array}$ & $\begin{array}{l}111 / 10 \\
<+1+\infty 1\end{array}$ & $\begin{array}{l}\mathbf{F} \\
\mathbf{P}\end{array}$ & آزمون آناليز واريانس با مشاهدات تكرارى \\
\hline
\end{tabular}

记 
جدول ب. ميانتين نمره توانمندى عضو فعال خانواده در بعد احساس خودكار آمدى در دو كروه آزمون و كنترل در زمانهاي مختلف

\begin{tabular}{|c|c|c|c|}
\hline \multicolumn{2}{|c|}{ آزمون t مسيثقل } & & \multirow{2}{*}{ زمان } \\
\hline $\mathbf{P}$ & $\mathrm{t}$ & & \\
\hline$V / \Delta$ & .188 & & قبل از مداخله \\
\hline$<+1++1$ & Ir/AT & & يكى هفته بعد از مداخله \\
\hline$<+/ *+1$ & $1 . / \pi^{2}$ & & يك ماه و نيم بعد از مداخله \\
\hline $\begin{array}{l}.1 . \Delta F \\
.19 \Delta\end{array}$ & $\begin{array}{l}8+1+* 4 \\
<+1 \cdot .1\end{array}$ & $\begin{array}{l}F \\
P\end{array}$ & آزمون آناليز واريانس با مشاهدات تكرارى \\
\hline
\end{tabular}

L

در هروهش حاضر نيز به نظر مىرسد با مداخله توانمندسازى

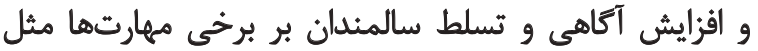

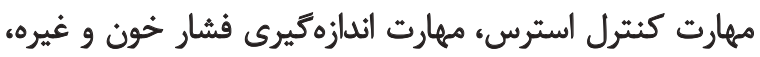

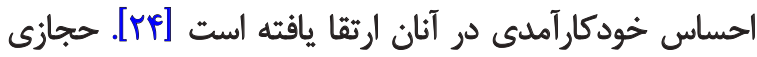

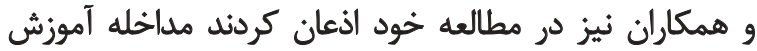

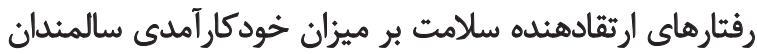

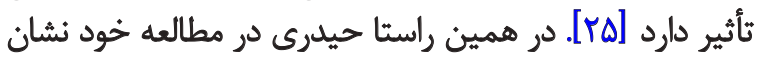

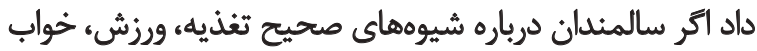

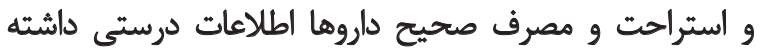

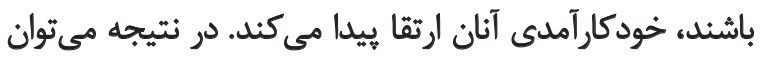

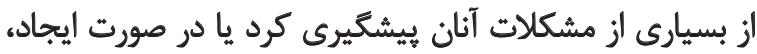

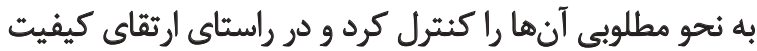

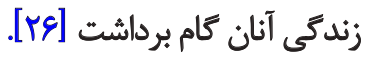

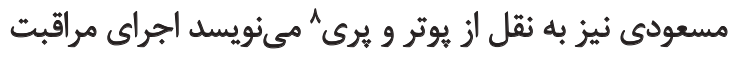

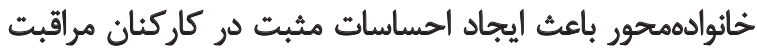

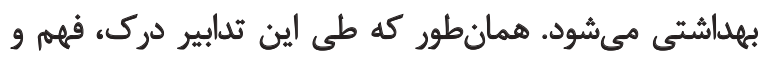

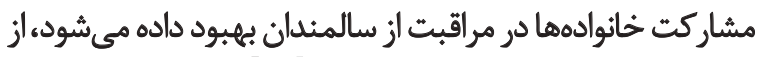

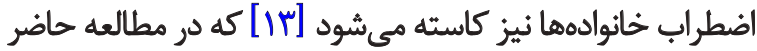

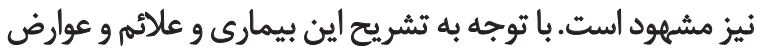

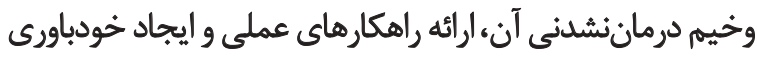

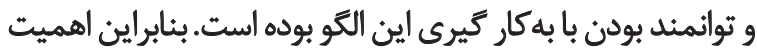

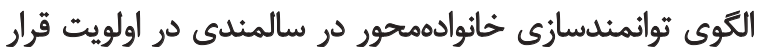

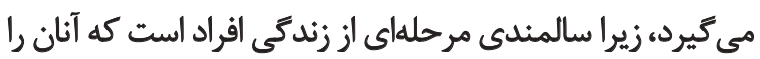

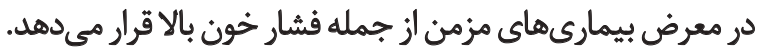

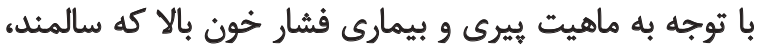

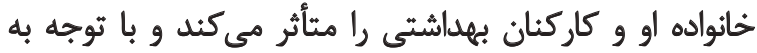

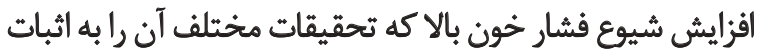

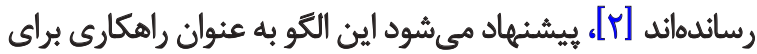

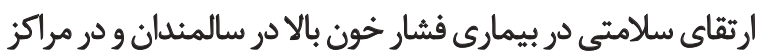
بهداشتى دمانى به كار ترفته شود.

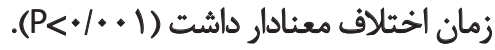

كنتايج اين بروهش نشان داد كه در بين دو كروه آزمون و و

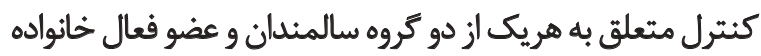

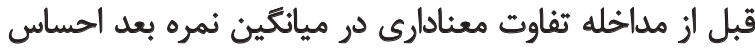

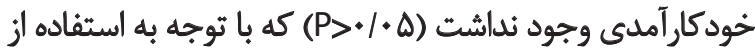

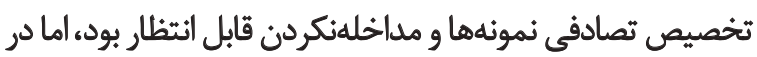

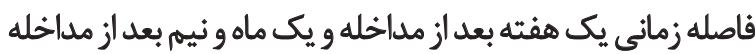

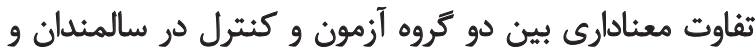

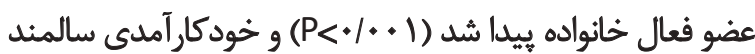

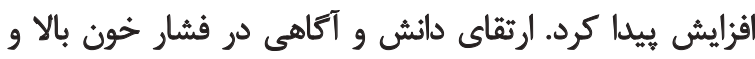

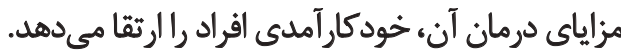

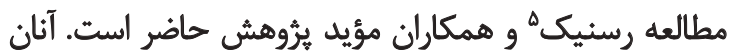

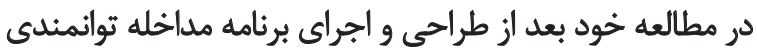

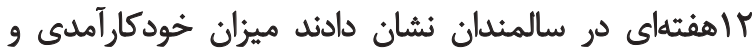

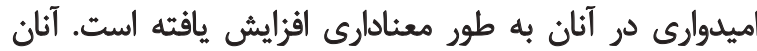

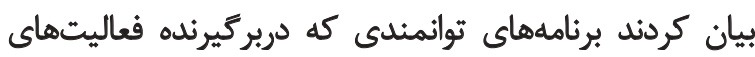

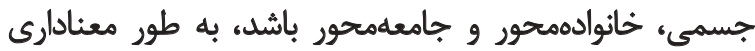

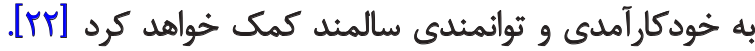

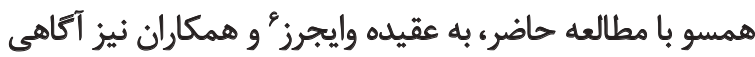

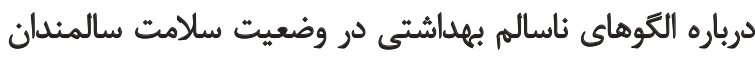

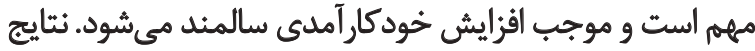

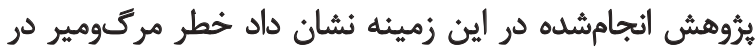

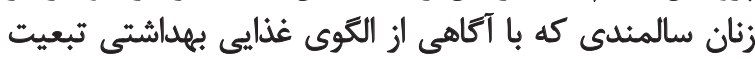

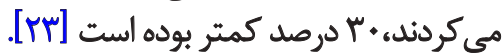

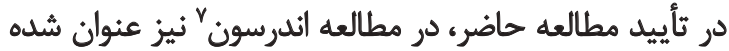

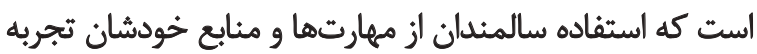

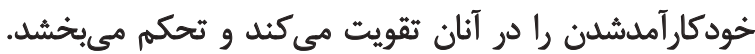


مطالعه حاضر نخستين مطالعه در بهكارتيرى التوى

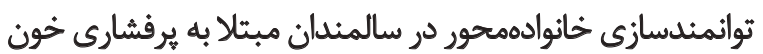

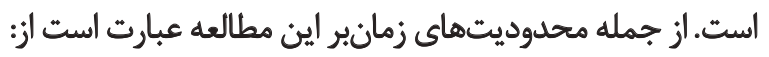

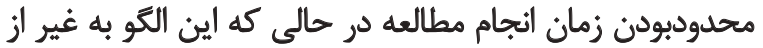

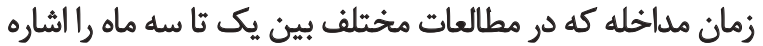

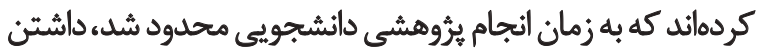

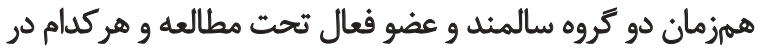

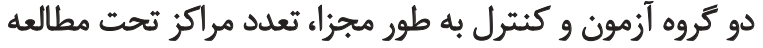

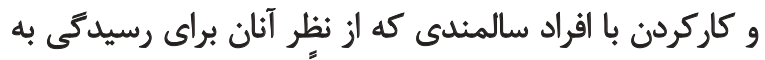

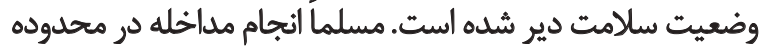

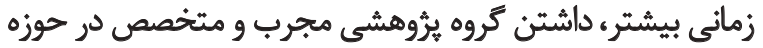

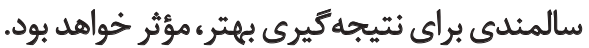

\section{نتيجلكيرى نمهايى}

با توجه به نتايج بروهش حاضر و اهميت خودكارآمدى در

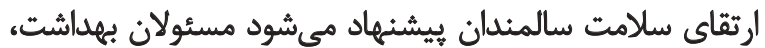

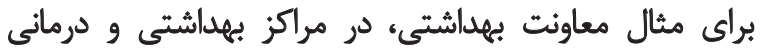

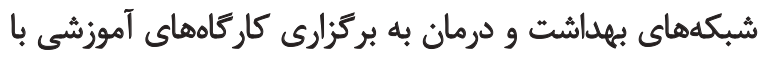

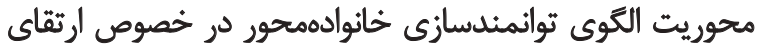

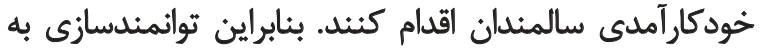

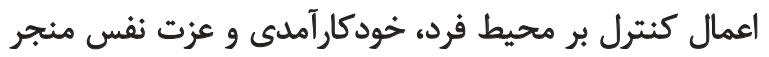

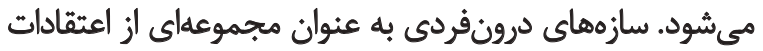

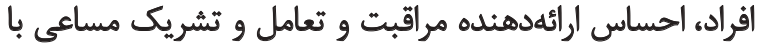

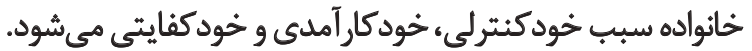

$$
\text { تشكر و قدردانى }
$$

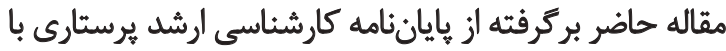

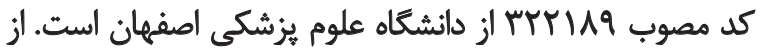

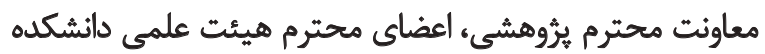

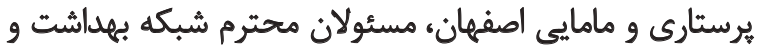

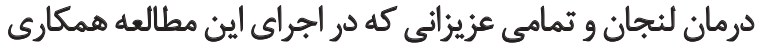

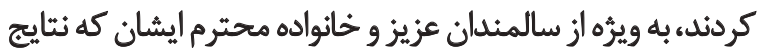

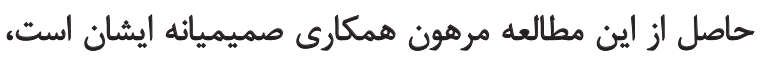

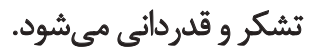




\section{References}

[1] Hojjati H, Koochaki G, Sanagoo A. [The relationship between loneliness and life satisfaction of the elderly in Gorgan and Gonbad cities (Persian)]. Journal of Research Development in Nursing \& Midwifery. 2012; 9(1):61-68.

[2] Rejeh N, Heravi Karimooi M, Montazeri A , Foroughan M. [Psychometric properties of the Iranian version of the facts on aging quiz (FAQI) (Persian)]. Payesh. 2012; 11(2):221-30

[3] Moeini B, Barati M, Jalilian F. [Factors associated with the functional independence level in older adults (Persian)]. Journal of Hormozgan University of Medical Sciences. 2012; 15(4):318-26.

[4] Nanbakhsh F, Mohaddesi H, Amirai A, Haji Shafiha M, Broomand F, Bahadori $\mathrm{F}$ et al. [The effect of health education on elderly women life quality (Persian)]. Journal of Payavard Salamat. 2011; 5(1):47-57.

[5] Chinnakali P, Mohan B, Upadhyay R, Singh A, Srivastava R, Yadav K. Hypertension in the elderly: Prevalence and health seeking behavior. North American Journal of Medical Sciences. 2012; 4(11):558-62. doi: 10.4103/1947-2714.103314

[6] Ahangari M, Kamali M, Arjmand Hesabi M. [The study of quality of life in the elderly with hypertension who are member of Tehran senile culture house clubs (Persian)]. Iranian Journal of Ageing. 2008; 3(1):26-32.

[7] Jalilian F, Emdadi S, Karimi M, Barati M, Gharibnavaz H. [Depression among collage students the role of general self-efficacy and perceived social support (Persian)]. Scientific Journal of Hamadan University of Medical Sciences. 2012; 18(4):60-6.

[8] Bandura A. Self-efficacy: Toward a unifying theory of behavioral change. Psychological Review. 1977; 84(2):191-215. doi: 10.1037/0033-295x.84.2.191

[9] Bernal H, Woolley S, Schensul JJ, Dickinson JK. Correlates of self-efficacy in diabetes self-care among Hispanic adults with diabetes. The Diabetes Educator. 2000; 26(4):673-80. doi: $10.1177 / 014572170002600415$

[10] Bandura A, Barbaranelli C, Caprara GV, Pastorelli C. Multifaceted impact of self-efficacy beliefs on academic functioning. child development. 1996; 67(3):1206-22. doi: 10.1111/j.1467-8624.1996. tb01791.x

[11] Allahyari I, Alhani F, Kazemnezhad A, Izadyar M. [The effect of family-centered empowerment model on the quality of life of school-age B-thalassemic children (Persian)]. Iranian Journal of Pediatrics. 2006; 16(4):455-61

[12] Abrahamian FM, DeBlieux PM, Emerman CL, Kollef MH, Kupersmith E, Leeper KV, et al. Health care-associated pneumonia: Identification and initial management in the ED. The American Journal of Emergency Medicine. 2008; 26(6):1-11. doi: 10.1016/j. ajem.2008.03.015

[13] Masoodi R, Alhani F, Moghadassi J, Ghorbani M. [The effect of family-centered empowerment model on skill, attitude, and knowledge of multiple sclerosis caregivers (Persian)]. Journal of Birjand University of Medical Sciences. 2010; 17(2):87-97.

[14] Stanhope M, Lancost J. Public Health Nursing. Missouri: Mosby; 2004

[15] Adams EK, Gavin NI, Ayadi MF, Santelli J, Raskind-Hood C. The costs of public services for teenage mothers post-welfare re- form: A ten-state study. Journal of Health Care Finance. 35(3):44 58. PMID: 19891207

[16] Alhani F. [Design and evaluation of the family-oriented prevention of iron deficiency Anemia (Persian)] [PhD dissertation] Tehran: Tarbiat Modares University; 2003.

[17] Masoudi R, Soleimani M, Qorbani M, Hasheminia A, Pour Dehkordi A, Bahrami N. [The effect of family centered empowerment model on the quality of life in elderly people (Persian)]. Journal of Qazvin University of Medical Sciences. 2010; 14(1):5764.

[18] Turnbull AP, Turbivill EV, Turnbull H. R. Evolution of family-professional partnerships: Collective empowerment as the model for the early twenty-first century. In: Shonkoff JP, Meisels SJ, Zigler EF, editors. Handbook of Early Childhood Intervention. Cambridge: Cambridge University Press; 2000

[19] Heydari S, Salahshourian-fard A, Rafii F, Hoseini F. [Correlation of perceived social support from different supportive sources and the size of social network with quality of life in cancer patients (Persian)]. Iran Journal of Nursing. 2009; 22(61):8-18.

[20] Taimori F, Alhani F, Kazemnezhad A. [The effect of familycentered empowerment model on the quality of life of school-age asthma children (Persian)]. Iranian Journal of Nursing Research. 2011; 6(20):52-63.

[21] Vahedian Azimi A, Alhani F, Ahmadi F, Kazemnejad A. Effec of family-oriented empowerment model on the life style of myocardial infarction patients. Iranian Journal of Critical Care Nursing. 2010; 2(4):127-32.

[22] Resnick B, Luisi D, Vogel A. Testing the Senior Exercise Selfefficacy Project (SESEP) for use with urban dwelling minority older adults. Public Health Nursing. 2008; 25(3):221-34. doi: 10.1111/j.1525-1446.2008.00699.x

[23] Waijers PM, Ocké MC, Van Rossum CT, Peeters PH, Bamia C, Chloptsios $Y$, et al. Dietary patterns and survival in older Dutch women. American Journal of Clinical Nutrition. 83(5):1170-6 PMID: 16685062

[24] Andersson M, Hallberg IR, Edberg A-K. Old people receiving municipal care, their experiences of what constitutes a good life in the last phase of life: A qualitative study. International Journal of Nursing Studies. 2008; 45(6):818-28. doi: 10.1016/j.ijnurstu.2007.04.003

[25] Hejazi S, Sahbaiee F, Fesharaki M, Abdollahi A. [The effect of education about health-promoting behaviors on the knowledge of the elderly in the geriatric nursing residences in Tehran (2009) (Persian)]. Journal of Birjand University of Medical Sciences. 2012; 19(1):114-21.

[26] Heidari M, Shahbazi S. [Effect of self-care training program on quality of life of elders (Persian)]. Iran Journal of Nursing. 2012 25(75):1-8. 
